\title{
Oxalate-Induced Photoreduction Dissolution and Transformation of Schwertmannite: Change of Mineral Phase and Elemental Fate-Supporting Information
}

Qian Yao ${ }^{\dagger}$, Chuling Guo ${ }^{*}+$, , Tingting Luo ${ }^{\S}$, Mengge Jiang ${ }^{\dagger}$, Xiaohu Jin ${ }^{\dagger}$, Xiaofei $\mathrm{Li}^{\dagger}$, Guining Lü, Xiaoyun $\mathrm{Yi}^{\dagger,+}$, Zhi Dang ${ }^{\dagger, *}$

$\uparrow$ School of Environment and Energy, South China University of Technology, Guangzhou, 510006, P. R. China

$¥$ The Key Laboratory of Pollution Control and Ecosystem Restoration in Industry Clusters, Ministry of Education, South China University of Technology, Guangzhou, 510006, P. R.

China

$\S$ School of Environmental Science and Engineering, Zhongkai University of Agriculture and Engineering, Guangzhou 510225, P. R. China

* Corresponding author:clguo@scut.edu.cn 
Figure S1 The sketch of the photo-reactor instrument

Figure S2 Variation of $\mathrm{pH}$ at the concentration of $0.5 \mathrm{mM}$ oxalate in the whole light and dark reaction 


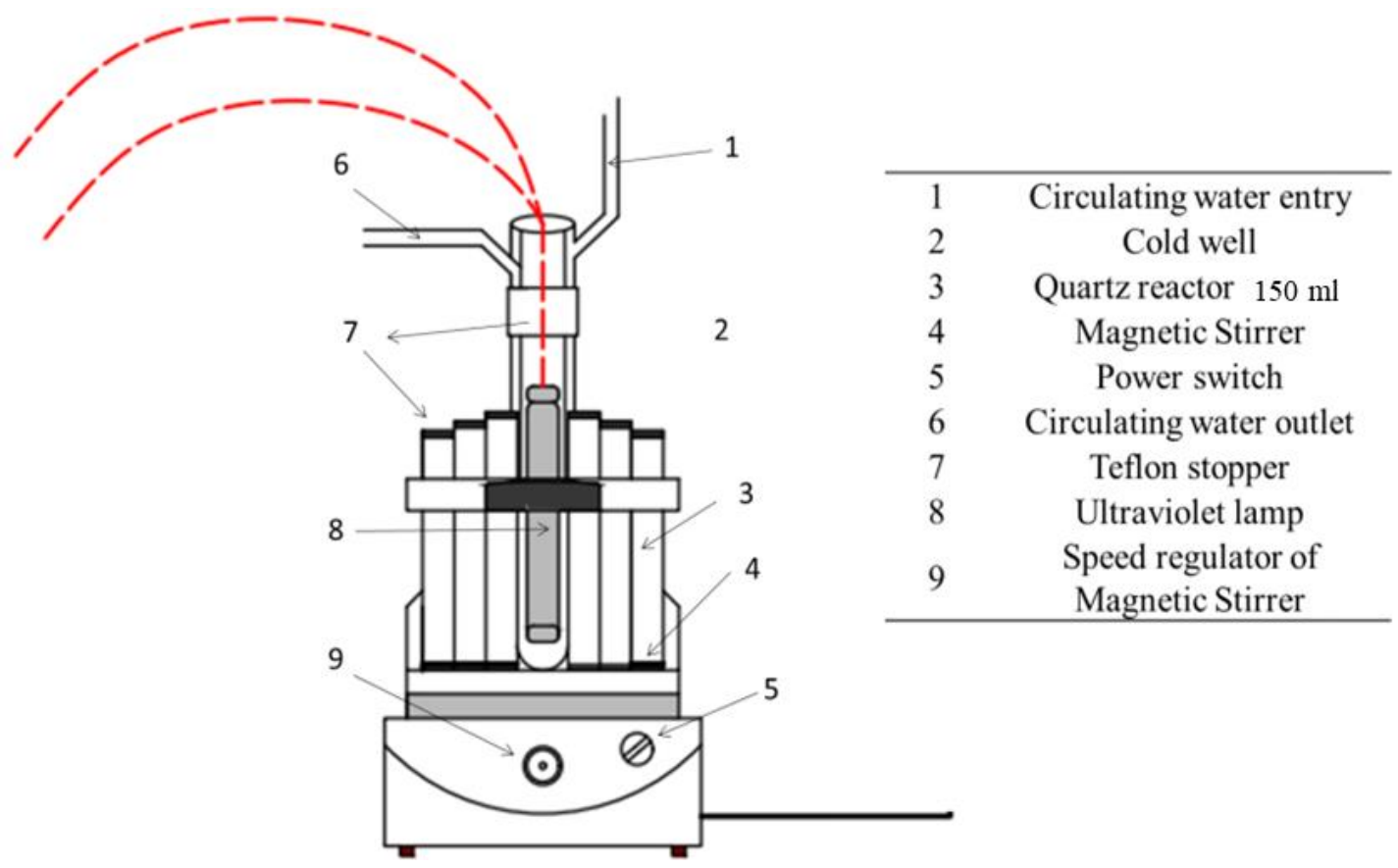

Figure S1. The sketch of the photo-reactor instrument. 


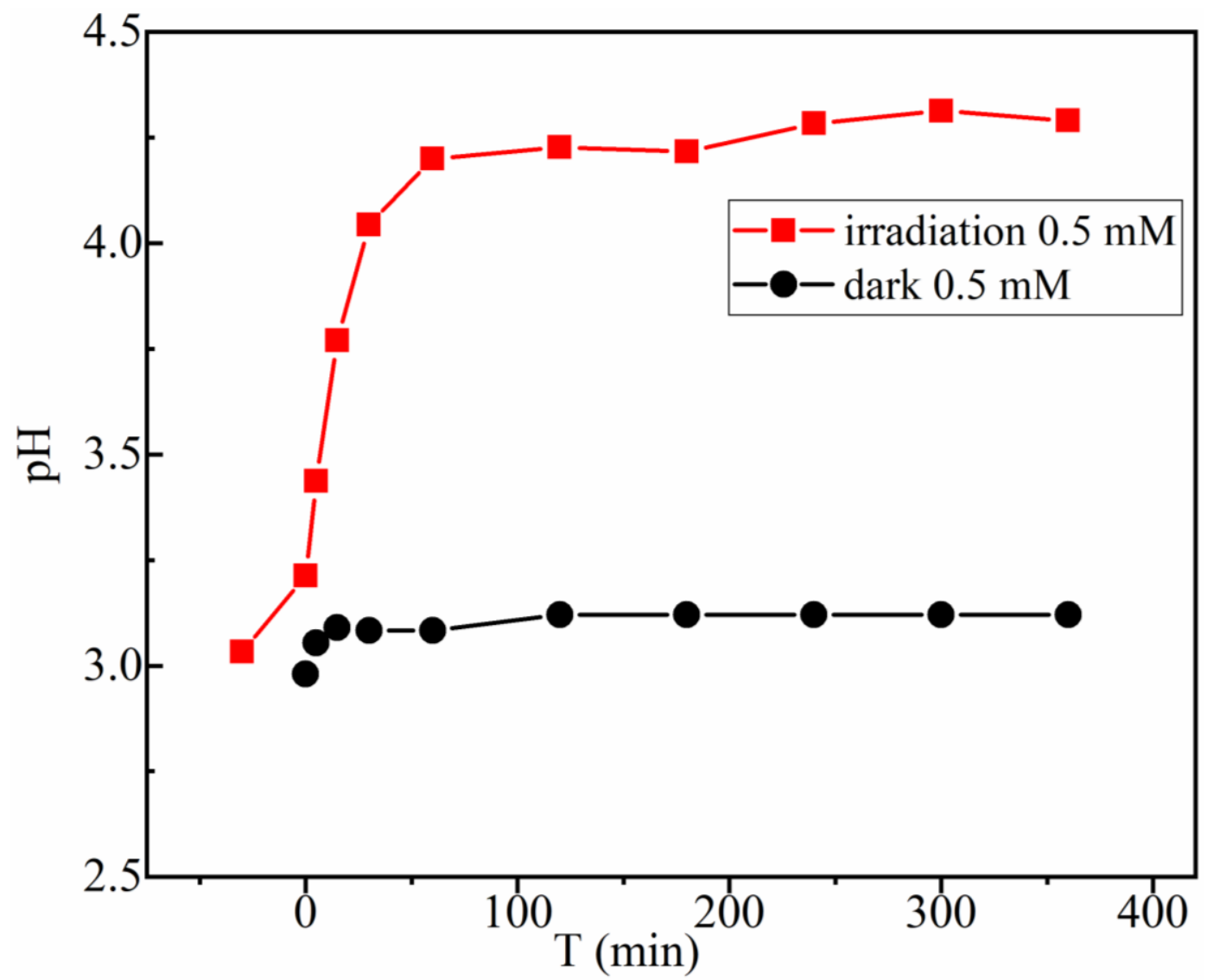

Figure $\mathrm{S} 2$. Variation of $\mathrm{pH}$ at the concentration of $0.5 \mathrm{mM}$ oxalate in the whole light and dark reaction. 